\title{
Néolithisation : À propos des noms berbères des céréales et des animaux domestiques. Note linguistique
}

\section{Salem Chaker}

\section{CpenEdition}

\section{Journals}

Édition électronique

URL : https://journals.openedition.org/encyclopedieberbere/2731

DOI : 10.4000/encyclopedieberbere.2731

ISSN : 2262-7197

Éditeur

Peeters Publishers

\section{Édition imprimée}

Date de publication : 5 octobre 2012

Pagination : 5520-5524

ISBN : 978-90-429-2718-6

ISSN : 1015-7344

\section{Référence électronique}

Salem Chaker, « Néolithisation : À propos des noms berbères des céréales et des animaux domestiques. Note linguistique », Encyclopédie berbère [En ligne], 34 | 2012, document N49, mis en ligne le 15 décembre 2020, consulté le 17 février 2022. URL : http://journals.openedition.org/ encyclopedieberbere/2731; DOI : https://doi.org/10.4000/encyclopedieberbere.2731

Ce document a été généré automatiquement le 17 février 2022.

(c) Tous droits réservés 


\title{
Néolithisation : À propos des noms berbères des céréales et des animaux domestiques. Note linguistique
}

\author{
Salem Chaker
}

1 Le très consistant dossier sur le processus de néolithisation en Afrique du Nord rassemblé dans ce volume amène le linguiste à se pencher à nouveau sur les dominations berbères de certains des paramètres qui fondent la notion même de néolithique : les céréales et les principaux animaux domestiques (mammifères), et leur propagation.

2 Le caractère fécond de l'étude des relations entre langue, culture et société a été abondamment et magistralement illustré par les travaux des grands savants indoeuropéanistes, notamment Emile Benveniste : ils montrent que l'étude fine et articulée de la morphogenèse et de la sémantogenèse de champs lexicaux centraux au plan socioculturel peut être extrêmement éclairante sur l'origine et la signification sociale première des pratiques humaines, qu'elles soient matérielles ou symboliques. La démarche est particulièrement intéressante dans un monde où les traces écrites sont rares et/ou tardives: la langue et les indices multiples qu'elle recèle constituent un outil essentiel pour apporter quelques lumières sur des configurations et pratiques sociales anciennes, proto-et préhistoriques.

Gabriel Camps, fondateur de cette encyclopédie, n'était pas linguiste, ni berbérisant au sens étroit du terme, mais il fut le premier à percevoir clairement (dès son Massinissa, 1961) tout ce que le préhistorien et protohistorien pouvaient tirer des données linguistiques berbères, sur le plan sociolinguistique bien sûr, mais surtout sur le plan de l'origine et de la diffusion des faits de culture et des pratiques sociales. Il a été le premier (1961, chap. II, notamment p. 77 à 80) à souligner le fait que si les Berbères actuels disposent, sur un territoire aussi vaste, du même vocabulaire pour dénommer 
les principales céréales, on est en droit de considérer cette donnée comme un indice fort de la grande ancienneté de la céréaliculture en Afrique du Nord:

«L'extension considérable de ce mot [irdən : blé], avec de très faibles variations [....] suffit à prouver non seulement l'ancienneté de la culture du blé dur en Berbérie, mais aussi l'association étroite de cette culture et des sociétés rurales berbères. » (p. 78).

Nous rajouterions volontiers : et, peut-être, de son caractère endogène, bien que cela n'ait pas encore été étayé.

Nous avons nous-même traité dans l'EB du vocabulaire berbère des grands animaux domestiques : «chèvre ", "chien", «cheval »... (cf. Bibl.). L'ensemble de ces études ciblées a été rassemblé dans un article de synthèse (Chaker 1995, repris et développé dans Chaker 1996, chap. 18).

Comme pour les céréales, il ressort de cette approche que, en dehors de quelques rares cas bien identifiables d'emprunts tardifs au latin - bœuf (de labour) : ayug, ayugu... - ou au sémitique à travers le latin - pour le chameau: alyem/aylam -, les termes fondamentaux qui désignent le chien, la chèvre, le mouton, la vache, le bœuf, le taureau, l'âne et sans doute le cheval, sont à la fois communs à l'ensemble du domaine berbère ( $=$ pan-berbères), de formation indigène (non empruntés) et très stables au plan sémantique.

7 Bien entendu, ces traits ne se déduisent pas de l'observation superficielle et immédiate des données synchroniques des dialectes actuels, mais reposent sur une analyse critique précise: a) des formes contemporaines et de leur genèse; b) de leur distribution géographique; c) de leurs variations sémantiques éventuelles à travers les dialectes. Ce n'est qu'au terme d'un tel examen que l'on est en droit de formuler des hypothèses reconstructives et historiques. A cet égard, on restera réservé devant les travaux (au moins depuis Greenberg) des linguistes généralistes ou " afro-asiatisants " dont le recours et le traitement « extensif » des données lexicales berbères n'obéissent pas à ces nécessités de méthode : toute reconstruction formelle ou sémantique et, a fortiori, toute comparaison avec des domaines linguistiques extérieurs, implique un travail préalable d'analyse critique et de reconstruction interne. On ne voit pas pourquoi les principes et méthodes qui ont permis de fonder la linguistique historique et comparée du domaine indo-européen ne s'appliqueraient pas ou s'appliqueraient avec moins de rigueur pour le berbère ou dans le domaine afro-asiatique.

8 Ces deux traits (pan-berbérité + stabilité sémantique du vocabulaire de base), mis en évidence tant pour les céréales que pour les grands animaux domestiques, autorisent indiscutablement à conclure à la grande ancienneté de ces deux pratiques agricoles chez les Berbères. Permettent-ils de conclure, comme nous sommes tentés de le faire, à leur caractère autochtone? - C'est une hypothèse que nous avons formulée (Chaker [1995]/1996, p. 270), et que nous soutiendrons ici, avec prudence et en nous gardant de toute généralisation. En effet, si sur un territoire aussi vaste, la langue berbère conserve un noyau lexical commun dans ces deux domaines de techniques agricoles fondamentales, c'est probablement que ce vocabulaire et les pratiques auxquelles il réfère se sont constitués en milieu berbère. On voit mal en effet, comment une telle communauté et stabilité du lexique se seraient maintenues si ces pratiques avaient été exogènes : a) les traces de l'emprunt culturel seraient encore perceptibles à travers la/ des traces d'emprunt linguistique; $b$ ) ce lexique serait sans doute plus variable si la pratique n'en avait été acquise que par diffusion à partir d'une source externe. En tout 
cas, la thèse de l'allochtonie de ces éléments cruciaux de la néolithisation ne s'impose pas au vu des matériaux linguistiques. Ce qui n'est pas le cas, par exemple, pour un autre domaine de pratiques technologiques - certes plus tardif -, la métallurgie : il est aisé de montrer que l'essentiel du matériel lexical de ce champ, bien que pan-berbère, est emprunté au sémitique (phénicien/punique) (cf. Chaker, M97, EB XXXI, 2010).

Certes l'hypothèse de l'autochtonie ne paraît pas, en l'état actuel des recherches, être confirmée par les données de la préhistoire et en particulier par la paléogénétique : les souches domestiques, végétales et animales, de l'Afrique du Nord méditerranéenne préhistorique étudiées ne sont pas indigènes mais proviennent du Proche et MoyenOrient ou de l'Europe du sud (Roubet N40; pour le Maroc, voir Ouchaou, N48; Ballouche, Ouchaou \& El Idrissi, N47), Mais par ailleurs, on identifie clairement une région de domestication ancienne des bovins dans le désert égypto-libyen et une zone de culture du mil/sorgho au Sahara, central (mais ce point reste discuté) et occidental (Mauritanie), indépendantes du Moyen-Orient, et à partir desquelles une diffusion s'est opérée vers l'Ouest/vers l'Est (Barich, N42; Ballouche, N41).

La situation est donc sans doute complexe et non-homogène pour l'ensemble de l'espace berbère et/ou selon les époques. Ce que, du reste, les données linguistiques elles-mêmes laissent entrevoir : comme nous l'avons formulé (Chaker 1995/1996), la coexistence pour certains animaux de plusieurs racines lexicales pan-berbères concurrentes : bovins : ${ }^{*} \mathrm{~S}(\mathrm{~W}),{ }^{*} \mathrm{ZGR},{ }^{*} \mathrm{FNS}$; ovin : KR(R) ; *WL ; *8S ; âne : YZD, rYL... pourrait être l'indice d'une diversité des espèces et même d'une chronologie relative entre les variétés ; ainsi, * $\mathrm{S}(\mathrm{W})$ (bovin) et YZD (âne) sont certainement plus anciens que, respectivement FNS (afunas) et YYL (ayyul), qui sont, au plan de leur morphogenèse, des adjectifs qualifiants, c'est-à-dire des dénominations secondaires de variétés particulières et/ou nouvelles.

Quoi qu'il en soit, on ne doit certainement pas tirer de conclusions hâtives, ni à partir des données linguistiques, ni à partir de données paléobotaniques et archéozoologiques encore très fragmentaires. C'est d'ailleurs bien ce que montre le cas du chien : son nom berbère (aydi/idan) est sans aucun doute, parmi les noms d'animaux domestiques, le plus stable et le plus commun au monde berbère; ce qui conduit à voir en lui, ici comme ailleurs, l'animal le plus anciennement domestiqué par l'homme (bien avant la néolithisation). Pourtant, les spécimens préhistoriques découverts ne semblent pas être issus d'une souche génétique locale (Ouchaou, N48). Mais les études en la matière ne font que commencer.

Pour ce qui est des végétaux, aux classiques irdən (blé), timz̧in/tumz̧in (orge), tażədkunt/ taz̧əkkunt (avoine), il convient de rajouter ilni/inəlli/enəle (mil, millet), dont la domestication est établie comme ancienne et locale au Sahara (central - ?) et occidental. Sous la notice «Mil» (M1 16b, EB XXXII), nous avons montré que les matériaux lexicaux confirment nettement cette ancienneté et même le statut d'« archétype » de la culture du mil/millet.

En l'état des connaissances, la prudence s'impose donc et il est assez probable que ces pratiques agricoles ont eu une genèse multiple et une diffusion complexe en Afrique du Nord, à partir de foyers locaux (plutôt sahariens), moyen-orientaux et ibériques.

La pan-berbérité et la stabilité sémantique de ce vocabulaire, qui sont incontestables, pourrait aussi être expliquées par une diffusion très rapide et ancienne à l'ensemble des communautés berbères de ce stock lexical de formation locale, quelle qu'ait été 
l'origine première de ces pratiques agricoles (diffusion à partir d'une source extérieure ou genèse locale). Ce qui n'aurait rien d'étonnant si l'on considère que la néolithisation fut une transformation comportementale profonde et continue.

On s'aventurera même à relier cette thématique à d'autres objets et pratiques sociales : le pastoralisme et le nomadisme* (voir les nombreux travaux de C. Roubet, notamment : N40, N45 et K51, K52, EB XXVII, 2005). On sera tenté de considérer comme $C$. Roubet que le néolithique nord-africain - et notamment celui des régions atlasiques - a été plus celui de pasteurs que d'agriculteurs ; pastoralisme néolithique qui a pu être le vecteur fondamental de la propagation des nouveaux comportements et techniques et du lexique qui y est associé. L'absence de terme commun pour désigner la maison en dur (cf. Chaker 2010 : « Maison... » M17c, EB XXX), la relative communauté et la grande précision des désignations de l'outre chez les Berbères actuels, nomades et sédentaires, (Chaker, 052), pourraient bien être la trace d'une culture pastorale (néolithique?) quasi-généralisée qui aurait permis la propagation rapide de ce vocabulaire commun d'un bout à l'autre de l'aire berbère.

Ce qui rejoint et confirmerait une hypothèse formulée par Blench (2001) qui a proposé d'expliquer la remarquable homogénéité du lexique berbère par une longue période de nomadisme qui aurait favorisé les échanges entre groupes et l'unité linguistique. On retrouve aussi une thèse très ancienne (homogénéité des parlers nomades / hétérogénéité des parlers sédentaires), formulée pour le berbère par André Basset (1938) qui soulignait la grande homogénéité phonétique des parlers berbères nomades (Touaregs) par opposition à l'infinie variation des parlers sédentaires; la thèse est confirmée pour une aire, l'Asie centrale, où coexistaient nomades et sédentaires par le grand linguiste Roman Jakobson (Basset 1938, cf. discussion).

Pour avoir une vue plus complète de cette problématique des liens potentiels entre techniques agricoles néolithiques et lexique berbère, il conviendra d'examiner ultérieurement le vocabulaire berbère de la poterie* puisque la céramique a été l'un trait décisif de la néolithisation en Afrique du Nord (cf. Roubet, N40, N45 \& 46 et Ballouche, Ouchaou \& El Idrissi, N47).

$18 \rightarrow$ Voir aussi 027. « Origines berbères : Linguistique et Préhistoire » (S. Chaker).

\section{BIBLIOGRAPHIE}

BASSET A., " Aires phonétiques homogènes et non homogènes ", Proceedings of the Third International Congress of Phonetic Sciences (Ghent), 1938, p. 258-261 (avec un commentaire de Roman Jakobson). Repris dans Articles de dialectologie berbère, Paris, Klincksieck, 1959, p. 29-32. BENVENISTE E., Problèmes de linguistique générale, I, Paris, NRF/Gallimard, 1966, notamment : Partie V. « Lexique et culture » et II, 1974, notamment : Partie V. « L'homme dans la langue ». BENVENISTE E., Le vocabulaire des institutions indo-européennes, I (Economie, parenté société) \& II (Pouvoir, droit, religion), Paris, Les Editions de Minuit, 1969. 
BLENCH R., "Types of language spread and their archaeological correlates : the example of Berber", Origini. Preistoria e Protoistoria delle Civiltà Antiche, XXIII, 2001, p. 169-189.

CAMPS G., Massinissa ou les débuts de l'histoire, Alger, 1961.

CHAKER S., « Linguistique et préhistoire : autour de quelques noms d'animaux domestiques en berbère ", L'homme méditerranéen. Mélanges offerts à Gabriel Camps, Aix, Publications de l'Université de Provence, 1995, p. 259-264. Repris et développé dans : Manuel de linguistique berbère II : Syntaxe et diachronie, Alger, ENAG, 1996, chap. 18.

CHAKER S., Encyclopédie berbère, notices relatives au vocabulaire des céréales, des animaux domestiques et de l'agriculture :

- fasc. IV, 1987 : « aliw, olivier », p. 529-530

- fasc. V, 1988 : « âne (dénominations berbères) », p. 656-657

- fasc. VIII, 1990 : «Azemmur (olivier)», p. 1220-1221

- fasc. XII, 1993 : « Cheval (dénominations berbères)», p. 1911

- fasc. XIII, 1994 : « Chèvre (dénominations berbères) », p. 1918 ; « Chien (dénominations berbères)», p. 1924

- fasc. XVII, 1996 : « Dromadaire (noms berbères du)», p. 2550-2554

- fasc. XVIII, 1997 : « Figuier/figues (nom berbère) », p. 2831-2833

- fasc. XXVI, 2004 : « Jujubier (Note linguistique)», p. 3981-3982

- fasc. XXVIII-XIX, 2008 : « Labour (note ling.) », p. 4311-4314 ; « Lait (note ling.) », p. 4330-4331 ;

« Lièvre-Lapin (note ling.) », p. 4414-4415

- fasc. XXX, 2010 : « Maison : Note linguistique complémentaire », p. 4528-4530

- fasc. XXXI, 2010 : « Meules et moulins (Noms berbères) » (avec K. Naït-Zerrad), p. 4959-4962

- fasc. XXXII, 2010 : « Mil : Note linguistique complémentaire », p. 5023-5024; « Mouton (sur le nom du)», p. 5096-5099.

- fasc. XXXIII, 2012 : «Olivier/Olive : Note linguistique complémentaire ».

LAOUST E., Mots et choses berbères, Paris, Challamel, 1920.

MEILLET A., Linguistique historique et linguistique générale, Paris, Champion, 1975.

\section{INDEX}

Mots-clés : Alimentation, Linguistique, Néolithique 